\title{
SEGURANÇA DO PACIENTE EM UNIDADE NEONATAL: PREOCUPAÇÕES E ESTRATÉGIAS VIVENCIADAS POR PAIS*
}

\author{
Fernanda Araujo Rodrigues ${ }^{1}$, Wiliam Wegner ${ }^{2}$, Karen Jeanne Cantarelli Kantorski ${ }^{3}$, Eva Neri Rubim Pedro ${ }^{4}$
}

RESUMO: Objetivo: analisar como os pais identificam a segurança do paciente em unidade neonatal. Método: estudo de caso exploratório, com abordagem qualitativa, realizado em hospital público de grande porte no sul do Brasil. Participaram do estudo 23 pais de crianças internadas na unidade neonatal, por meio de entrevista semiestruturada, no período entre junho e agosto de 2015. Realizou-se análise de conteúdo do tipo temática. Resultados: emergiram duas categorias: "Preocupações com a Segurança do Paciente" e "Estratégias para a Segurança do Paciente". Os familiares relataram preocupações relacionadas com o controle de acesso à unidade, risco de infecção e comunicação deficiente com a equipe de saúde. No entanto, percebem-se excelência no serviço prestado, prática de cuidado com amor e ambiente protegido. Conclusão: apesar de inquietações, os pais sentem-se seguros em relação à assistência, considerando que houve mais estratégias identificadas do que preocupações.

DESCRITORES: Segurança do Paciente; Criança Hospitalizada; Enfermagem Neonatal; Pais.

\section{PATIENT SAFETY IN A NEONATAL UNIT: CONCERNS AND STRATEGIES EXPERIENCED BY PARENTS}

\begin{abstract}
Objective: to analyze how parents identify patient safety in a neonatal unit. Method: this was a qualitative exploratory case study carried out in a large public hospital in the South of Brazil. Participants included 23 parents of children hospitalized in the neonatal unit, using semi-structured interviews, between June and August 2015. The data were submitted to thematic content analysis. Results: two categories emerged: "Concerns with Patient Safety" and "Strategies for Patient Safety." Family members reported concerns relative to access control to the unit, risk of infection and flawed communication among health team members. However, excellence of the care provided, loving care practices, and a protected environment were observed. Conclusion: despite some concerns, parents felt safe about the care provided, expressing more safety strategies than problems.
\end{abstract}

DESCRITORES: Patient Safety; Hospitalized Child; Neonatal Nursing; Parents.

\section{SEGURIDAD DEL PACIENTE EN UNIDAD NEONATAL: PREOCUPACIONES Y ESTRATEGIAS EXPERIMENTADAS POR PADRES}

RESUMEN: Objetivo: analizar de qué modo los padres identifican la seguridad del paciente en unidad neonatal. Método: estudio de caso exploratorio con abordaje cualitativo, realizado en hospital público de gran porte en el Sur de Brasil. Participaron del estudio 23 padres de niños internados en la unidad neonatal, mediante entrevista semiestructurada, en el período entre junio y agosto de 2015. Se aplicó análisis de contenido de tipo temático. Resultados: surgieron dos categorías: “Preocupaciones por la Seguridad del Paciente" y "Estrategias para la Seguridad del Paciente". Los familiares informaron preocupaciones relacionadas con el control de acceso a la unidad, riesgo de infección y comunicación deficiente con el equipo de salud. No obstante, se percibió excelencia en el servicio brindado, práctica de atención con amor y ambiente protegido. Conclusión: a pesar de sus inquietudes, los padres se sienten seguros en relación a la atención, considerando que hubo más estrategias identificadas que preocupaciones.

DESCRIPTORES: Seguridad del Paciente; Niño Hospitalizado; Enfermería Neonatal; Padres.

*Artigo extraído da dissertação intitulada: Acompanhantes de Crianças Hospitalizadas em Unidade Neonatal: preocupações e estratégias relacionadas à segurança do paciente. Universidade de Federal do Rio Grande do Sul, 2016.

${ }^{1}$ Enfermeira. Mestre em Enfermagem. Especialista em Saúde na Secretaria Estadual de Saúde. Porto Alegre, RS, Brasil. ${ }^{2}$ Enfermeiro. Doutor em Enfermagem. Professor Adjunto da Escola de Enfermagem da Universidade Federal do Rio Grande do Sul. Porto Alegre, RS, Brasil.

${ }^{3}$ Enfermeira. Mestre em Enfermagem. Doutoranda em Enfermagem na Universidade Federal do Rio Grande do Sul. Porto Alegre, RS, Brasil.

${ }^{4}$ Enfermeira. Doutora em Educação. Professora Titular da Escola de Enfermagem da Universidade Federal do Rio Grande do Sul. Porto Alegre, RS, Brasil. 


\section{INTRODUÇÃO}

Em Unidade de Internação Neonatal (UIN) há distintas circunstâncias que permeiam riscos às crianças; por isso, incidentes de segurança, com ou sem danos, podem ocorrer independente da intenção dos profissionais. Ressalta-se que um incidente consiste emum evento que pode resultar em dano desnecessário; e, evento adverso, um incidente que resultou em dano ${ }^{(1)}$. Assim, estabelece-se como segurança do paciente a redução do risco de danos desnecessários associados aos cuidados de saúde para um mínimo aceitável ${ }^{(1)}$.

As circunstâncias de cuidado caracterizam o atendimento ofertado, sendo tal concepção proposta para além de intervenções, envolvendo equipe de saúde, paciente, familiar, contexto ecenário ${ }^{(2)}$. Os incidentes de segurança nas UIN relacionam-se, principalmente, com o processo medicamentoso, sendo suas causas associadas a fatores humanos ${ }^{(3)}$. O manuseio por vários profissionais e o prolongado período de internação constituem elementos que elevam a exposição à falha ${ }^{(3)}$.

A inserção do pai e/ou da mãe na rotina beneficia paciente, familiar e instituição. Porém, apesar de delegar cuidado aos pais, percebe-se a família apenas como um acompanhante que auxilia quando convém aos profissionais ${ }^{(4)}$. No entanto, a assistência em neonatologia deve extrapolar a criança, envolvendo também o cuidado com o familiar ${ }^{(4)}$.

A presença do acompanhante consiste em requisito fundamental para o desenvolvimento da cultura de segurança, pois as mães possuem atitudes proativas como: capacidade de observar, oferecer proteção à criança e questionar os profissionais ${ }^{(2)}$. Portanto, salienta-se a importância de a equipe conhecer os sentimentos do familiar em relação à situação experenciada.

Acredita-se que, de forma geral, acompanhantes podem ser parceiros para a segurança na identificação e prevenção de eventos adversos. Ainda, o conhecimento da perspectiva da família pode contribuir para a mudança do cenário de segurança do paciente ${ }^{(5)}$. Assim, diante da escassez de estudos sobre incidentes/eventos adversos em UIN ${ }^{(3)}$ e do pressuposto de que a participação do familiar não é explorada em sua plenitude ${ }^{(6)}$, justifica-se a realização desta pesquisa, aqual teve como questão norteadora: Quais as preocupações e estratégias sobrea segurança do paciente percebidas por pais em unidade neonatal? Portanto, este estudo teve por objetivo analisar como ospais identificam a segurança do paciente em unidade neonatal.

\section{MÉTODO}

Estudo de caso exploratório, com abordagem qualitativa. Este possui finalidade de desenvolver hipóteses e proposições pertinentes à investigação inicial ${ }^{(7)}$. Definiu-se como caso:A vivência dos pais da criança hospitalizada em UIN relacionadas com a segurança do paciente.

O cenáriofoi a UIN de um hospital público de grande porte na região sul do Brasil, queestá organizada em oito enfermarias: vinte (20) leitos de cuidado intermediário convencional, dez (10) de cuidado intermediário canguru e vinte (20) de terapia intensiva. A instituição é certificada pela Joint CommissionInternational(JCI) e desenvolve política de gerenciamento de riscos e de segurança do paciente, incluindo notificação, avaliação e promoção de melhoriasde incidentes e riscos, visando sua prevenção.

Os participantes do estudo foram 23 familiares, sendo 16 mães e sete pais. Estabeleceram-se como critérios de inclusão: ser pai/mãe de criança hospitalizada entre cinco e 50 dias; possuir idade maior ou igual a 18 anos, e apresentar condições emocionais/psicológicas para relatar sua vivência. E, critérios de exclusão: ser pai/mãe de criança que sofreu tentativa de aborto intencional; não visitar o filho todos os dias ou permanecer menos de uma hora por dia na UIN.

A coleta das informações ocorreu entre junho e agosto de 2015, por meio de entrevista semiestruturada que contemplava questões relativas à internação e à segurança do paciente. Definiuse o número de participantes conforme o critério de saturação ${ }^{(8)}$. As entrevistas foram realizadas por uma única pesquisadoraem sala reservada eexterna à UIN, com duração média de 20 minutos. 
As informações obtidas foram submetidas à técnica de análise de conteúdo do tipo temática, a qual busca atribuir significados a partir dos núcleos de sentido ${ }^{(8)}$. Utilizou-se o software NVivo versão 10 para organização das informações.

Os preceitos éticos foram contemplados e o projeto foi aprovado pelo Comitê de Ética (Parecer n. ${ }^{\circ}$ 1.094.423). Para garantir o anonimato, os participantes foram codificados por letras, seguidos por números, de acordo com a ordem das entrevistas. Utilizou-se a letra " $M$ " para mães e a letra " $P$ " para pais.

\section{RESULTADOS}

A partir da análise das informações, emergiram duas categorias temáticas: "Preocupações com a Segurança do Paciente" e "Estratégias para a Segurança do Paciente". A seguir, apresentam-se categorias e subcategorias.

\section{Preocupações com a Segurança do Paciente}

O processamento das informações permitiu identificar 95 unidades de significado relacionadas com as situações que foram consideradascomo possíveis riscos à segurança da criança. A análise identificou que 16 participantes mencionaram pelo menos uma preocupação, independente da circunstância de cuidado. Assim, emergiram cinco subcategorias que contemplaram aspectos sobre falta de controle de visitas, insuficiência de informações, problemas quanto às rotinas da unidade e da dinâmica da equipe, falhas na realização de cuidados, e risco de infecção.

Na subcategoria "Falta um pouco de controle na entrada",o acesso à unidade foi relatado como preocupação para a segurança da criança por dois participantes. As declarações exemplificam:

Mas o simples fato de tu chegar e apertar a campainha e ter alguém do outro lado para te atender.(P2)

Falta um pouco de controle é na questão das visitas [...] Isso aí que eu fiquei mais preocupada.(M20)

Quatropais identificaram a comunicação como fator de preocupação com a segurança, constituindose a subcategoria "Informação na teoria". As falas evidenciam essa questão:

Quando a gente entra na UTI neonatal tem escrito: Os pais têm acesso à informação [...] Mas tem, realmente, na teoria; na prática, não é o que acontece.(P2)

Falta isso, de todo o dia o médico vir falar[...] Eu acho que eles usam termos muito técnicos.(M13)

Aspectos relacionados com a dinâmica da equipe de saúde e com as rotinas da unidade foram elencados como preocupações por cinco participantes, emergindo a subcategoria "A gente encontra um pouquinho de dificuldade". Os depoimentos confirmam esse aspecto:

A gente encontra um pouquinho mais de dificuldade porque trocou a equipe.(M1)

Final de semana, por exemplo, para a gente é um tempo perdido, né? Eu digo porque o planejamento da equipe médica se limita muito de segunda à sexta.(P2)

Aquela que satura, das máquinas que apitam aquelas de remédio, tudo que apita, para mim, é um tormento. (M7)

A subcategoria "Deu alguma coisa colateral" evidenciou a preocupação de oito participantes frente à assistência à criança hospitalizada. Os depoimentos confirmam:

E o [nome do bebê] pelado e ela jogando água em cima, dando banho [...] Tu imaginas o grau de insegurança. (M1)

Imaginas, tu ir para casa sabendo que estão fazendo um exame que é desnecessário.(P2) 
Eles deixaram o cordão umbilical dele durante nove dias, né? Ele desenvolveu um trombo.(P11)

Para mim, ele fez com uma ignorância [...] Que o caninho que estava dentro do pulmão dela soltou.(P12)

É, a fixação que, às vezes, fica pertinho do olho [...] e isso que eu fico com medo.(M14)

A subcategoria "O bebê entra sem problema e depois..." está relacionada com o risco de infecção. Dois pais verbalizaram tais preocupações:

Às vezes, o bebê entra sem problema algum e acaba adquirindo uma bactéria.(M8)

Tem pai que entra e sai com os aventais, vão para dentro dos quartos, não lavam as mãos.(M9)

\section{Estratégias para a Segurança do Paciente}

No processamento das informações foram identificadas196 unidades de significado relacionadas com asações implementadas, com o objetivo de garantir um cuidado seguro, sendo que todos os pais referiram, pelo menos, uma estratégia.Constitui-se por seis subcategorias, que abordam questões sobre controle de acesso à unidade, comunicação efetiva, serviço de excelência, cuidado com empatia, precauções para evitar infecção, avaliação do risco de queda, adequada identificação do paciente, e medidas para a correta prescrição/administração de medicamentos.

Na subcategoria "Aqui não é lugar de visita", o acesso dos pais e/ou visitantes apareceuna fala de sete participantes, porém, agora como estratégia para a segurança. Os trechos ilustram esse ponto:

Eu posso não estar aqui, mas eu sei que não vai entrar outra pessoa.(M3)

Esse horário [de visita] de meia hora também, onde não tem muita movimentação de pessoas, né? Para proteger cada paciente.(P11)

Não poder entrar com celular [...]é importante porque eu não queria foto dele divulgada.(M20)

Na subcategoria"Tudo é muito bem conversado",15participantes relataram a comunicação efetiva como estratégia, seja entre equipe de saúde e familiar ou entre os próprios profissionais. As declarações exemplificam esse aspecto:

Tudo é muito bem conversado[...]e mesmo assim passado para mim.(M1)

Ele [médico] nos passa aquela segurança, aquela coisa que todo o pai e toda a mãe quer ouvir.(M7)

Questões como a estrutura, o quantitativo adequado de profissionais e o padrão de atendimento foram citadas por 12 participantes como significativas à segurança da criança na subcategoria "Prestação de serviço com excelência". Os depoimentos evidenciam isso:

Só em ter esse banco de leite aqui é uma outra história.(M7)

Ele [bebê] está pelo plano [privado], mas os coleguinhas dele não estão e o atendimento é o mesmo.(P11)

Elas [enfermeiras] vão até retirando ele [bebê] dos locais onde está mais grave para uma sala mais intermediária.(M16)

Na subcategoria "Cuidado com amor", 20 participantes descreveram a realização de cuidados com empatia como estratégia para a segurança da criança hospitalizada, como exemplificado:

Fazem como se fosse filho delas, né? Como uma mãe.(P7)

Não cuidam só como profissional, elas cuidam com amor.(M20)

Ainda nessa subcategoria, observou-se que a identificação do paciente, apesar de ser uma metaa ser alcançada no processo de acreditação e considerando que a instituição é reconhecida pela $\mathrm{JCl}$,foi citada como estratégia por apenas duas participantes, sendo que uma delas afirmou:

Quem não chama pelo nome não cuida. (M7) 
Outras circunstâncias estão relacionadas com a prevenção de infecção, como citadas por oito participantes. A partir delas, construiu-se a subcategoria "Tem que lavar as mãos",na qual as declarações contextualizam esse aspecto:

O termômetro ser de cada um, de cada um ter as suas coisinhas.(M13)

Tem que lavar as mãos, tem que se higienizar.(M20)

Na subcategoria "Ambiente protegido", quatro participantes descreveram a avaliação do risco de queda e a implementação de medidas para evitar esse desfecho como estratégias, de acordo com os relatos:

Elas [técnicas de enfermagem] vão pesar ele, elas enrolam ele em um pano [...]as portas da incubadora estão sempre fechadas.(M7)

\section{DISCUSSÃO}

Em relação ao acesso, na subcategoria "Falta um pouco de controle na entrada",pertencente à primeira categoria, percebeu-se a importância conferida à disponibilidade de um profissional para a recepção. A presença de um secretário é apresentada como sugestão na literatura ${ }^{(9)}$. No entanto, na UIN pesquisada, esse profissional está disponível de modo contínuo durante o horário comercial; nos demais períodos, ele divide sua carga horária entre outras unidades, sendo necessário acioná-lo por telefone.

Neste contexto, destaca-se a responsabilidade compartilhada, visto que na falta de um profissional, outros podem assumir sua tarefa em prol da segurança do paciente. No entanto, tal responsabilidade ainda não é compartilhada igualmente entre os membros da equipe ${ }^{(10)}$.

Sobre um profissional em tempo integral na recepção, identificou-se uma importante preocupação com o fluxo de pessoas. Assim, a forma como ocorre o acesso está relacionada com o acolhimento, sendo que essa prática permite avançar no aspecto da qualidade do serviço, contemplando os anseios dos familiares, as orientações preconizadas pelas políticas de saúde e pelos órgãos de certificação de qualidade ${ }^{(11)}$.

Ainda sobre acesso, ressalta-se que, nesta UIN, mãe e pai têm acesso liberado nas 24 horas do dia. Para outras pessoas, há um horário pré-determinado de 30 minutos, com a indicação dos pais sobre os visitantes permitidos.

Quanto à subcategoria "Aqui não é lugar de visita", pertencente à segunda categoria,os pais aprovam, de modo geral, o regramento quanto ao acesso à unidade. Há controle desde a recepção central da instituição, onde deve ser apresentado documento para adquirir um crachá, até a porta da UIN, que permanece fechada, sendo aberta após identificação via interfone.

$\mathrm{Na}$ subcategoria "Informação na teoria",algunsparticipantes identificaram a comunicação insuficiente como uma preocupação. No entanto, é dever dos profissionais minimizar esse quadro. Tal achado corrobora um estudoque identificou que a equipe multiprofissional pode reduzir tais consequências, por meio da escuta qualificada das demandas do acompanhante e com a disponibilização de informações ${ }^{(12)}$.

Em contrapartida, na subcategoria "Tudo é muito bem conversado",a maioria dos participantes valoriza o diálogo e se sente segura, referindo tranquilidade para ir para a casa. Tal fato corrobora que a transmissão de informações diminui o nível de ansiedade dos pais e aumentam seu contentamento ${ }^{(13)}$.

Outro estudo considerou a comunicação entre equipe e familiar como uma forma de orientar cuidados no âmbito domiciliar, espaço no qual os pais não possuirão auxílio(2). Em consonância, esta pesquisa identificou por meio de algumas declarações, orientações quanto à segurança da criança.

Atualmente, ainda existe uma mudança importante e potencialmente transformadora relacionada com o papel dos familiares, sendo que a comunicação efetiva por meio de informações sobre diagnóstico e tratamento favorece o envolvimento dos pais nos cuidados dos filhos ${ }^{(14)}$. 
Nesse contexto, o enfermeiro apresenta fundamental importância, pois ao não se utilizar de uma comunicação eficiente, passa a ser compreendido como um profissional meramente técnico, deixando uma lacuna no cuidado ${ }^{(12)}$. Ressalta-se que a comunicação deficiente contribui para a angústia dos pais, especialmente, quando não são consultados ou informados sobre procedimentos ou intercorrências ${ }^{(15)}$.

Diante da subcategoria "A gente encontra um pouquinho de dificuldade",observou-se que, assim como em outra pesquisa, os participantes consideram olocal pouco acolhedor, apresentam preocupação com o rodízio entre as equipes, necessitando adaptar-se ao ambiente rico em tecnologia, com diversos ruídos, o que pode desencadear medo ${ }^{(12)}$.

Nessa perspectiva, a utilização dos equipamentos pode estar relacionada com eventos adversos. São considerados riscos relacionados com os equipamentos médico-hospitalares:defeito no aparelho, erro de utilização, uso em circunstâncias impróprias e falta de manutenção preventiva(16).

Ainda sobre a dinâmica da equipe e a rotina da unidade, na subcategoria "Prestação de serviço com excelência", a estrutura, que se dá por enfermarias de acordo com o quadro clínico; o Banco de Leite Humano (BLH); e o número de profissionais foram citados como elementos que subsidiam a segurança. A importância do BLH relaciona-se com o fato que durante a hospitalização, as mães compreendem que o modo de auxiliar na recuperação é através do leite, sendo este uma forma natural de proteção ${ }^{(13)}$.

Sobre o quantitativo de profissionais, esse foi valorizado, tendo em vista que os pacientes são atendidos equitativamente em todos os turnos de trabalho, ao contrário do observado em pesquisa semelhante, onde o número insuficiente foi uma reclamação ${ }^{(2)}$. $\mathrm{O}$ dimensionamento adequado em relação à carga de trabalho tem associação direta com a qualidade do cuidado ${ }^{(17)} \mathrm{e}$, consequentemente, com a segurança.

A subcategoria "Deu alguma coisa colateral"surgiu do receio em relação com alguns cuidados. Os exemplos foram: controle da temperatura corporal e formação de trombos, a partir da permanência prolongada de cateter umbilical.

Os pais identificaram os riscos em conformidade com a literatura. A trombogênese é incomum nos primeiros cinco dias de inserção, sendo que, após esse período, há uma predisposição à trombose portal; e este tipo de dano está associado a fatorescomo pré-eclâmpsia e prematuridade ${ }^{(18)}$, como no caso do paciente desta pesquisa.

Ainda sobre falhas no cuidado, surgiu a preocupação com extubação. Em determinados serviços, a aspiração segue critérios baseados na experiência do profissional, o que maximiza o risco de complicações ${ }^{(19)}$. Há outras possíveis falhas relacionadas com a extubação acidental. Uma pesquisa indicou que $38 \%$ dos casos estão associados à obstrução por secreção, configurando aspiração insuficiente ${ }^{(20)}$. Outro estudo correlacionou com a realização de radiografias, sem o devido cuidado ${ }^{(21)}$.

Uma participante ainda destacouo receio quanto às possíveis lesões decorrentes da fixação do CPAP (Continuous Positive AirwayPressure). Nesse sentido, a dimensão da pronga e o método inadequado de fixação são aspectos potencializadores para lesões ${ }^{(22)}$. Cabe destacar que, nesta UIN, utiliza-se curativo de hidrocolóide com compressa aluminizada para minimizar o atrito entre pronga e septo nasal. Em relação à fixação, aplica-se o mesmo curativo de hidrocolóide nas regiões de contato com o elástico.

Ampliando a discussão sobre a assistência, a partir da subcategoria "Cuidado com amor",é possível inferir o reconhecimento da humanização da assistência. Neste contexto, a equipe de enfermagem possui função primordial. O exercício da enfermagem deve estar centralizado no cuidado, que é respaldado pelo conhecimento, pela construção de relações adequadas e embasado em habilidades para a promoção de um ambiente seguro(17).

A despeito da relevância sobre identificação do paciente, percebeu-se fragilidade nessa rotina, sendo reconhecida por apenas duas participantes. Porém, visando tal reconhecimento, a instituição elaborou um Plano de Identificação dos Pacientes, que estabelece critérios para a correta identificação. Entendese que a identificação é de responsabilidade multidisciplinar, envolvendo aspectos de estrutura, processos de trabalho, cultura organizacional, prática profissional e participação do usuário ${ }^{(23)}$. 
Outra circunstância que apareceu como estratégia na fala de apenas duas participantes, foi aadministração segura de medicamentos. A terapia medicamentosa envolve diversas etapas: prescrição/ aprazamento, preparo, administração e monitoramento de ação/reação ao fármaco ${ }^{(24)}$. A UIN apresenta prescrição informatizada. Na prescrição médica consta nome do medicamento, apresentação e posologia; enquanto na prescrição de enfermagem, encontra-se o cálculo para diluição, considerando as doses fracionadas.

Sobre a relevância do enfermeiro nesse processo, ressalta-se que, apesar de se preconizar a realização de técnicas mais complexas por enfermeiros, na prática percebe-se que são atribuídas iguais atividades a enfermeiros e técnicos de enfermagem ${ }^{(24)}$. No entanto, tal situação não se confirmou na UIN, onde a realização de alguns procedimentos é de responsabilidade exclusiva do enfermeiro, tais como o preparo de medicamentos intravenosos como a prostaglandina E1.

Ainda se recomenda a utilização de bombas de infusão para maior controle do gotejamento e a dispensação de doses unitárias, as quais apresentam rotulagem com informações completas ${ }^{(24-25)}$. Tais situações foram observadas nesta pesquisa.

A subcategoria "O bebê entra sem problema e depois..." aborda a preocupação com o risco de infecções. De forma semelhante, outro estudo evidenciou que os pais observaram que visitantes e funcionários nem sempre seguiam boas práticas para a prevenção ${ }^{(15)}$. A infecção é um processo multifatorial e tem como possíveis causas:estrutura física, dimensionamento insuficiente dos profissionais, superlotação e descumprimento das normas dehigienização ${ }^{(26)}$. Ainda sobre infecções, na subcategoria "Tem que lavar as mãos", destacou-se a restrição ao toque no paciente. Tal método está previsto em publicação que sugere o manuseio mínimo, a fim de evitar manipulações desnecessárias ${ }^{(27)}$. Isso pode ser considerado uma estratégia para promoção da segurança do paciente na atenção neonatal.

A utilização de insumos descartáveis e de materiais individuais também foi citada como estratégia. Além desses fatores, outras precauções relacionadas com os cuidados estão associadas ao controle de infecção, como a rotina de troca do circuito do respirador, o registro da data de realização de curativo e da abertura de equipos. Todas essas ações são rotinas na UIN pesquisada.

Por fim, na subcategoria "Ambiente protegido",surgiu a atenção dos profissionais, especialmente técnicos de enfermagem, ante o risco de queda. Tal fato pode ser justificado pela cultura de segurança constituída com o Protocolo Assistencial de Quedas em Crianças,elaborado pela instituição, que contribui para a sistematização do cuidado e qualidade assistencial.

A UIN encontra-se entre os serviços de risco, motivo pelo qual os pacientes não utilizavam pulseira de identificação diferenciada. Ainda sobre o risco de quedas, a prevenção deve ser um cuidado compartilhado entre equipe e família, configurando um elemento para a segurança da criança ${ }^{(2)}$.

As declarações sobre o risco de queda foram compatíveis com as recomendações da literatura: manter rodas de berço/incubadora travadas, assim como portas da incubadora; envolver o paciente em cueiro para pesar e dar banho; e realizar prescrição de enfermagem contemplando tais cuidados $^{(2)}$.

Dentre as limitações, destaca-se a dificuldade de realização das entrevistas, considerando a complexidade do tema. Verifica-se que ainda há muito a ser estudado sobre o assunto; e, por tratar-se de um estudo de caso qualitativo, a análise é representativa deste contexto. Contudo, seria interessante reproduzir a investigação em outros estabelecimentos, visando contemplar diferentes cenários.

\section{CONSIDERAÇÕES FINAIS}

Ao analisar como ospais identificamasegurança da criança hospitalizada em UIN, este estudo desvelou que, apesar de algumas inquietações, essesse sentem seguros, considerando que houve uma quantidade significativamente maior de referências que contemplem situações classificadas como estratégias do que em comparação às preocupações. Esse fato denota a característica positiva da instituição que, como certificada pela $\mathrm{JCl}$, evidencia a segurança do paciente como prioridade. 
A pesquisa evidenciou questões como problemas relacionados com as rotinas da unidade e da equipe assistencial e falhas de cuidados. Além disso, apontou caminhos para qualificar a prática profissional. Para tanto, sugere-se prezar pela comunicação efetiva, por meio de espaços permanentes para diálogo entre equipe assistencial e familiar; bem como manter o quantitativo adequado de profissionais, visando a realização satisfatória da assistência, como avaliação da pele, administração segura de medicamento e cuidados relacionados com a ventilação mecânica.

A análise de como a equipe de saúde reconhece/valoriza a atuação dos pais durante a hospitalização se configura como um direcionamento para a construção do cuidado seguro, visto que a busca pela segurança do paciente exige empenho mútuo.

Salientam-se, como principais contribuições desta pesquisa, o reconhecimento dospais e da responsabilidade a eles atribuída durante a hospitalização; a possível qualificação da assistência, a partir da adoção de condutas em conformidade com as estratégias descritas; e, consequentemente, o desenvolvimento de uma cultura de segurança.

\section{REFERÊNCIAS}

1. World Health Organization. More Than Words: Conceptual Framework for the International Classification for Patient Safety. [Internet] Genebra: World Health Organization; 2009 [acessoem 05 abr 2015]. Disponível: http:// www.who.int/patientsafety/taxonomy/icps_full_report.pdf.

2. Wegner W, Pedro ENR. A segurança do paciente nas circunstâncias de cuidado: prevenção de eventos adversos na hospitalização infantil. Rev. Latino-Am. Enfermagem. [Internet] 2012;20(3)[acesso em 10 mar 2015]. Disponível:http://dx.doi.org/10.1590/S0104-11692012000300002.

3. Lanzillotti LS, de Seta MH, de Andrade CLT, Mendes Junior WV.Eventos adversos e outros incidentes na unidade de terapia intensiva neonatal. Ciênc. saúde coletiva. [Internet]2015;20(3) [acesso em 20 mar 2015]. Disponível:http://dx.doi.org/10.1590/1413-81232015203.16912013.

4. Silva TRG, Manzo BF, Fioreti FCCF, Silva PM. Cuidado centrado na família na perspectiva de enfermeiras da Unidade de Terapia Intensiva Neonatal. Rev Rene. [Internet] 2016;17(5) [acesso em 13dez 2016]. Disponível:http:// dx.doi.org/10.15253/2175-6783.2016000500009.

5. Arab M, Akbari Sari A, MovahedKor E, Hosseini M,TolouiRakhshanS, EzatiM. Patient Safety in Tehran University of Medical Sciences' General Hospitals, Iran. Iran J PublicHealth. [Internet] 2013;42(3) [acesso em 13 dez 2016]. Disponível: https://www.ncbi.nlm.nih.gov/pmc/articles/PMC3633801.

6. Dittz ES, de Sena RR, Motta JAC, Duarte ED.Cuidado materno ao recém-nascido na unidade de terapia intensiva neonatal: possibilidades e desafio. Cienc. enferm.[Internet] 2011;17(1) [acesso em 28 fev 2015]. Disponível: http:// dx.doi.org/10.4067/S0717-95532011000100006.

7. Yin RK. Estudo de caso: planejamento e métodos. $4^{\mathrm{a}}$ ed. Porto Alegre: Bookman; 2010.

8. Minayo MCS. O desafio do conhecimento: pesquisa qualitativa em saúde. 12a ed. São Paulo: Hucitec; 2010.

9. Tamez RN. Enfermagem em UTI neonatal: assistência ao recém-nascido de alto risco. $5^{\text {a }}$ ed. Rio de Janeiro: Guanabara Koogan; 2013.

10. Bohomol E, Tartali JA. Eventos adversos em pacientes cirúrgicos: conhecimento dos profissionais de enfermagem. Actapaul.enferm. [Internet] 2013;26(4) [acesso em 25 set 2015]. Disponível:http://dx.doi.org/10.1590/ S0103-21002013000400012.

11. Sanches ICP, Couto IRR, Abrahão AL, Andrade M. Acompanhamento hospitalar: direito ou concessão ao usuário hospitalizado?Ciênc. Saúde Colet. [Internet] 2013;18(1) [acesso em 28 dez 2014]. Disponível: http://dx.doi. org/10.1590/S1413-81232013000100008. 
12. Rodrigues EN, Oliveira ERC, Julião AMS. Assistência em unidade de terapia intensiva pediátrica: percepção do acompanhante. R. Interd. [Internet] 2014;7(4) [acesso em 28 fev 2015]. Disponível: https://revistainterdisciplinar. uninovafapi.edu.br/index.php/revinter/article/view/492.

13. Frigo J, Zocche DAA, Palavro GL, Turatti LA, Neves ET, Schaefer TM. Percepções de pais de recém-nascidos prematuros em unidade de terapia intensive neonatal. RevEnferm UFSM. [Internet] 2015;5(1) [acesso em 13 nov 2015]. Disponível: http://dx.doi.org/10.5902/2179769212900.

14. Carman KL, Dardess P, Maurer M, Sofaer S, Adams K, Bechtel C, et al. Patient and family engagement: a framework for understanding the elements and developing interventions and policies. HealthAffairs. [Internet] 2013;32(2) [acessoem 12 abr 2017]. Disponível: http://dx.doi.org/10.1377/hlthaff.2012.1133.

15. Lyndon A, Jacobson CH, Fagan KM,Wisner K, Franck LS.Parents' perspectiveson safety in neonatal intensivecare: a mixed-methodsstudy.BMJ QualSaf. [Internet] 2014;23(11) [acesso em 03fev 2017]. Disponível:http://dx.doi. org/10.1136/bmjqs-2014-003009.

16. Lima HO, Dutra ECR. O Gerenciamento de Riscos na Saúde - aplicação na atenção hospitalar. Revista de Administração Hospitalar e Inovação em Saúde. [Internet] 2010;(5) [acesso em 16 out 2016]. Disponível:http:// dx.doi.org/10.21450/rahis.v0i5.1114.

17. Cavalcante AKCB, Rocha RC, Nogueira LT, Avelino FVSD, da Rocha SS. Cuidado seguro ao paciente: contribuições da enfermagem. Rev Cubanaenfermer. [Internet] 2015;31(4) [acesso em 30 dez 2015]. Disponível: http://www.revenfermeria.sld.cu/index.php/enf/article/view/907.

18. Kido RYZ, Alvares BR, Mezzacappa MAMS. Umbilical catheters in newborns: indications, complications and imaging diagnosis. ScientiaMedica. [Internet] 2015;25(1) [acesso em 16mar 2016]. Disponível:http://dx.doi. org/10.15448/1980-6108.2015.1.19236.

19. Gonçalves RL, Tsuzuki LM, Carvalho MGS. Aspiração endotraqueal em recém-nascidos intubados: uma revisão integrativa da literatura. Rev.bras. ter. intensiva. [Internet] 2015;27(3) [acesso em 25 out 2015]. Disponível: http://dx.doi.org/10.5935/0103-507X.20150048.

20. Ventura CMU, Alves JGB, Meneses JA. Eventos adversos em Unidade de Terapia Intensiva Neonatal. Rev. bras. enferm. [Internet] 2012;65(1) [acesso em 23 nov 2015]. Disponível: http://dx.doi.org/10.1590/S003471672012000100007.

21. Duarte SCM, Queiroz ABA, Büscher A, Stipp MAC. O erro humano no cotidiano da assistência de enfermagem em terapia intensiva. Rev. Latino-Am. Enfermagem. [Internet] 2015;23(6) [acesso em 04 jan 2016]. Disponível: http://dx.doi.org/10.1590/0104-1169.0479.2651.

22. Nunes CR, de Castro SB, da Motta GCP, da Silva AM, Shardosim JM, da Cunha MLC. Método de prevenção de lesão nasal causada por CPAP em recém-nascido pré-termo: relato de caso. Revista HCPA. [Internet] 2012;32(4) [acesso em 22 out 2015]. Disponível: http://seer.ufrgs.br/hcpa/article/view/35004.

23. Tase TH, Lourenção DCA, Bianchini SM, Tronchin DMR. Identificação do Paciente nas organizações de saúde: uma reflexão emergente. Rev. Gaúcha Enferm. [Internet] 2013;34(3) [acesso em 25 nov 2015]. Disponível: http:// dx.doi.org/10.1590/S1983-14472013000300025.

24. Harada MJCS, Chanes DC, Kusahara DM, Pedreira MLG. Segurança na administração de medicamentos em Pediatria. Acta paul.enferm. [Internet] 2012;25(4) [acesso em 17 nov 2015]. Disponível: http://dx.doi.org/10.1590/ S0103-21002012000400025.

25. Alves KYA, da Costa TD, de Barros AG, de Lima KYN, Santos VEP. Segurança do paciente na terapia intravenosa em unidade de terapia intensiva. Revista de Pesquisa Cuidado é Fundamental. [Internet] 2016;8(1) [acesso em 10 jan 2017]. Disponível: http://dx.doi.org/10.9789/2175-5361.2016.v8i1.3714-3724.

26. Shimabukuro PMS, Ferreira MR, Feldman LB. A gestão e o impacto da higiene hospitalar no serviço de neonatologia. JInfectControl. [Internet] 2015;4(1) [acesso em 24 jul 2015]. Disponível: http://jic.abih.net.br/index. $\mathrm{php/jic/article/view/79.}$

27. Bañuelos LGS, Gutiérrez JP, Velázques FET, Rubio MD. Seguridaddel neonato hospitalizado. Aproximaciones y propuestas. EnfermeríaUniversitaria. [Internet] 2012;9(2) [acesso em 09 mar 2015]. Disponível: http://www.scielo. org.mx/pdf/eu/v9n2/v9n2a4.pdf. 